\title{
A longitudinal study of CEACAM1 expression in melanoma disease progression
}

\author{
DOUGLAS ZIPPEL ${ }^{1,2^{*}}$, HANI BARLEV $^{1 *}$, RONA ORTENBERG $^{3}$, IRIS BARSHACK $^{2,4}$, \\ JACOB SCHACHTER $^{2,3^{* *}}$ and GAL MARKEL ${ }^{2,3,5^{* *}}$ \\ ${ }^{1}$ Department of Surgery C, Chaim Sheba Medical Center, Tel Hashomer; ${ }^{2}$ Sackler Faculty of Medicine, \\ Tel Aviv University, Tel Aviv; ${ }^{3}$ Ella Institute of Melanoma, ${ }^{4}$ Institute of Pathology and \\ ${ }^{5}$ Talpiot Medical Leadership Program, Chaim Sheba Medical Center, Tel Hashomer, Israel
}

Received October 1, 2014; Accepted November 24, 2014

DOI: $10.3892 /$ or.2014.3703

\begin{abstract}
The present study characterized the carcinoembryonic antigen-related cell adhesion molecule 1 (CEACAM1) expression profile in a longitudinal study during melanoma progression, in lesions obtained from the same patients: a primary skin lesion, a lymph node and a distant metastasis. The present study is expected to increase our understanding of the expression patterns of CEACAM1 in melanoma development. We identified 20 patients who could be analyzed for CEACAM1 expression over the course of disease progression. The pathology blocks were cut, and two slides were generated for each specimen. One underwent standard hematoxylin and eosin (H\&E) staining and a corresponding slide underwent immunohistochemical staining for the detection of CEACAM1. For 13 patients who were able to be followed up serially from primary lesion, lymph node and distant metastasis, a borderline significant increase in the staining of the membrane was noted $(\mathrm{P}=0.06)$. In contrast, there was no equivalent increase in cytoplasmic CEACAM1 in the same group of patients. For the cohort of 20 patients with primary and distant metastasis, a significant increase in the membrane staining was noted $(\mathrm{P}=0.026)$ and again, no equivalent significant increase in cytoplasmic staining was observed. We report that CEACAM1 expression increases along the course of disease development and progression of a patient. CEACAM1 represents a novel area of research which may have profound influence in future methods of harnessing cellular immunity to combat this disease. The results of the present study confirm that CEACAM1 is potentially an extremely useful target in arresting melanoma progression.
\end{abstract}

Correspondence to: Dr Douglas Zippel, Department of Surgery C, Chaim Sheba Medical Center, Tel Hashomer 52621, Israel

E-mail: dougbz@gmail.com

Dr Gal Markel, Sackler Faculty of Medicine, Tel Aviv University, Tel Aviv, Israel

E-mail: markel@post.tau.ac.il

${ }^{*, * *}$ Contributed equally

Key words: CEACAM1, metastatic melanoma, immunotherapy

\section{Introduction}

Malignant melanoma is highly immunogenic and responds to immunotherapy (1). As expected, promising results in metastatic melanoma have been observed with anti-CTLA-4 $(2,3)$, anti-PD-1 (4), anti-PD-L1 (5) and combinations. However, as the disease still progresses in many of the patients, additional modalities are needed.Clinical and biologic evidence highlights carcinoembryonic antigen-related cell adhesion molecule 1 (CEACAM1) as a possible target for immunotherapy (6). CEACAM1 is a transmembranal glycoprotein belonging to the Ig superfamily, composed of several extracellular Ig-like domains (7). CEACAM1 has been shown to be involved in the development of cancerous transformation, particularly in melanoma, yet also in non-small cell lung cancer and gastric carcinoma (8-10). Importantly, a strong correlation between CEACAM1 expression and the development of metastatic disease with poor overall survival was demonstrated in melanoma (11) and in lung cancer $(12,13)$. CEACAM1 operates by homophilic intercellular interactions with another CEACAM1 molecule. Engagement of CEACAM1 expressed by activated lymphocytes causes downstream signaling resulting in dephosphorylation of tyrosine residues, which consequently inhibits effector functions $(14,15)$. Indeed, CEACAM1 was shown to protect melanoma cells by inhibiting the action of NK and activated T cells $(6,16-18)$. This may be part of a sophisticated method of metastatic melanoma cells escaping the inherent immunogenic response which we see prevalent in melanoma. It has also been found that serum levels of circulating CEACAM1 correlate with response to established immunotherapy treatment (19).

Most of the currently available information on CEACAM1 expression in melanoma is derived from comparisons between patients and healthy individuals, or between isolated samples from various patients. There is to date, no data on CEACAM1 expression in a cohort of patients followed up from the primary tumor, lymphatic spread and subsequent distant metastasis. The present study characterized the CEACAM1 expression profile in a longitudinal study during melanoma progression, in lesions obtained from the same patients: a primary skin lesion, a lymph node and a distant metastasis. The present study is therefore expected to increase our understanding of 
Table I. Pathological features of the primary melanoma lesions.

\begin{tabular}{|c|c|c|c|c|c|}
\hline Pts & Clark & Breslow & Ulceration & $\begin{array}{l}\text { Vascular } \\
\text { invasion }\end{array}$ & $\begin{array}{l}\text { Lymphocyte } \\
\text { infiltration }\end{array}$ \\
\hline 1 & 4 & 3.8 & No & No & No \\
\hline 2 & 5 & 3.1 & No & No & No \\
\hline 3 & 2 & 1.2 & No & No & Yes \\
\hline 4 & N/A & N/A & No & No & No \\
\hline 5 & 3 & 1.84 & No & No & No \\
\hline 6 & 2 & 0.5 & No & No & No \\
\hline 7 & 3 & 1.8 & No & No & Yes \\
\hline 8 & 5 & 4 & Yes & No & No \\
\hline 9 & 5 & 30 & Yes & No & No \\
\hline 10 & 5 & 6 & Yes & No & No \\
\hline 11 & 5 & 5 & No & No & No \\
\hline 12 & 5 & 3.5 & No & No & No \\
\hline 13 & 5 & 5 & No & No & No \\
\hline 14 & 5 & 3.5 & Yes & No & Yes \\
\hline 15 & 5 & 5 & Yes & No & No \\
\hline 16 & 5 & 14 & No & No & No \\
\hline 17 & 4 & 2.75 & No & No & No \\
\hline 18 & 5 & 15 & No & No & Yes \\
\hline 19 & 5 & 6 & No & No & No \\
\hline 20 & 2 & 1.4 & No & No & No \\
\hline
\end{tabular}

Pts, patients; N/A, not available. ment. We also compared the expression of CEACAM1 in different metastatic lesions obtained from the same patients. Since CEACAM1 is a potential target for immunotherapy, this pilot study will provide initial data on the variability of CEACAM1 expression in potential target lesions.

\section{Materials and methods}

Patients. The Ella Institute for Melanoma, within the Chaim Sheba Medical Center, maintains a prospective database of melanoma patients and is the largest tertiary referral center for melanoma in Israel. From our database we searched and identified 13 patients with pathology paraffin block specimens available in our institution which included a primary lesion, a subsequent lymph node metastasis and finally a distant metastasis. In addition we found 7 patients with a primary lesion and subsequent distant metastasis, for a total of 20 patients who were analyzed for CEACAM1 expression over the course of disease progression. In addition, we analyzed 4 patients who had pathology specimens of metastases from more than one site. We also identified 5 patients who, in addition to a primary invasive lesion, had another lesion with melanoma in situ for comparison. All studies were conducted according to the approval of the Institutional Review Board.

Pathology and immunohistochemistry. The pathology blocks were cut, and two slides were generated for each specimen. One underwent standard hematoxylin and eosin (H\&E) staining and a corresponding slide underwent immunohistochemical staining for the detection of CEACAM1, as previously described in detail by Ortenberg et al (6). Briefly, the slides were prepared for immunostaining using standard protocols and were incubated overnight at $4^{\circ} \mathrm{C}$ with MRG1 mAb, which is a murine IgG1 monoclonal antibody against human CEACAM1. It recognizes the CEACAM1-specific $\mathrm{N}$-domain with high selectivity and affinity $\left(K_{\mathrm{D}} \sim 2 \mathrm{nmol} / \mathrm{l}\right)$. Detection was conducted using the Histostain-SP Broad Spectrum kit (Invitrogen, Carlsbad, CA, USA) and visualized with the substrate chromogen AEC. Sections were counterstained with hematoxylin (Ventana Medical Systems, Tucson, AZ, USA) and coverslipped with an aqueous mounting fluid (glycergel). Stained sections were reviewed by an expert pathologist, and suitable digital images were captured with an Olympus BX51 microscope. Slides were assessed for both membranal and cytoplasmic staining. The percentage of cells stained and the intensity of staining on a scale of $0-3$ were noted and recorded. In addition, primary lesions were assessed for Clark level, Breslow depth, presence of ulceration and lymphocytic infiltration.

Statistical analysis. Statistical analyses using standard non-parametric testing, including Kruskal-Wallis one-way analysis of variance, Friedman's two-way analysis of variance, Mann-Whitney-Wilcoxon test, Spearman's rank correlation coefficient, as well as Fisher's exact test, were performed on the data by an independent biostatistician.

\section{Results}

After reviewing the database and pathology specimens of all patients undergoing treatment for melanoma in the Chaim Sheba Medical Center, 29 pathology specimens were identified which were included in the present study.

We identified the following groups of patients and i) 13 patients with pathology specimens including a primary invasive lesion, a lymph node and a distant metastasis; ii) 7 patients with pathology specimens including a primary invasive lesion and a distant metastasis; iii) 4 patients with pathology specimens with multiple metastatic lesions; and iv) 5 patients with melanoma in situ, who were also evaluated for CEACAM1 membranal and cytoplasmic staining, were included.

The pathological features of the primary lesions are summarized in Table I.

Melanoma in situ preparations had no evidence of any membranal or cytoplasmic CEACAM1.

For the invasive primary lesions, staining intensity was correlated directly and significantly with the percentage of cells stained, in both membranal CEACAM1 $(\mathrm{P}<0.001)$ and cytoplasmic CEACAM1 (P<0.02) (Fig. 1).

However, no correlation was found between the amount of membranal CEACAM1 staining and Clark level or Breslow depth of the primary lesions (Fig. 2). For the 13 patients who were able to be followed up serially from primary lesion, lymph node and distant metastasis, a borderline significant increase in membranal staining was noted (Kruskal-Wallis test, $\mathrm{P}=0.06)$. In contrast, there was no equivalent increase in cytoplasmic CEACAM1 in the same group of patients (Fig. 3). 

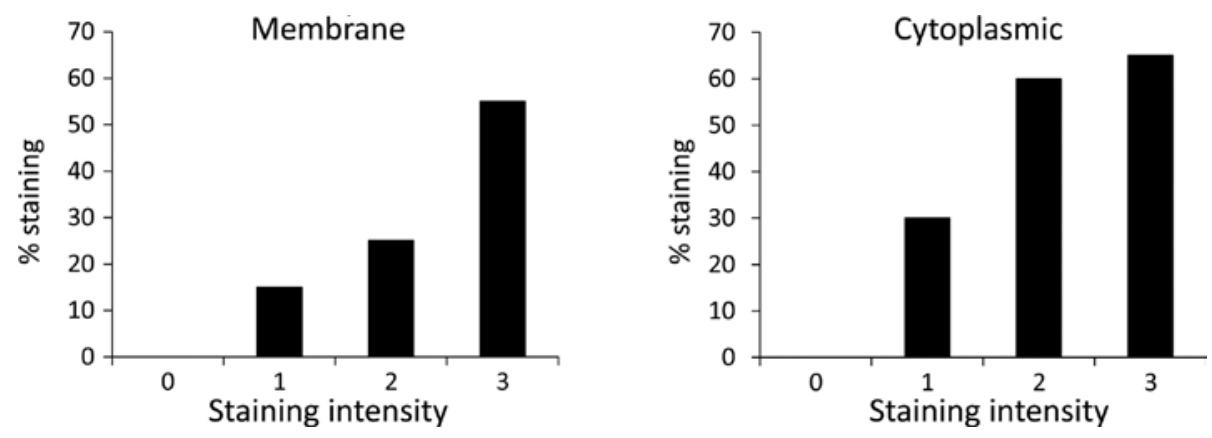

Figure 1. Staining intensity vs. percentage of cells stained, membrane and cytoplasm.
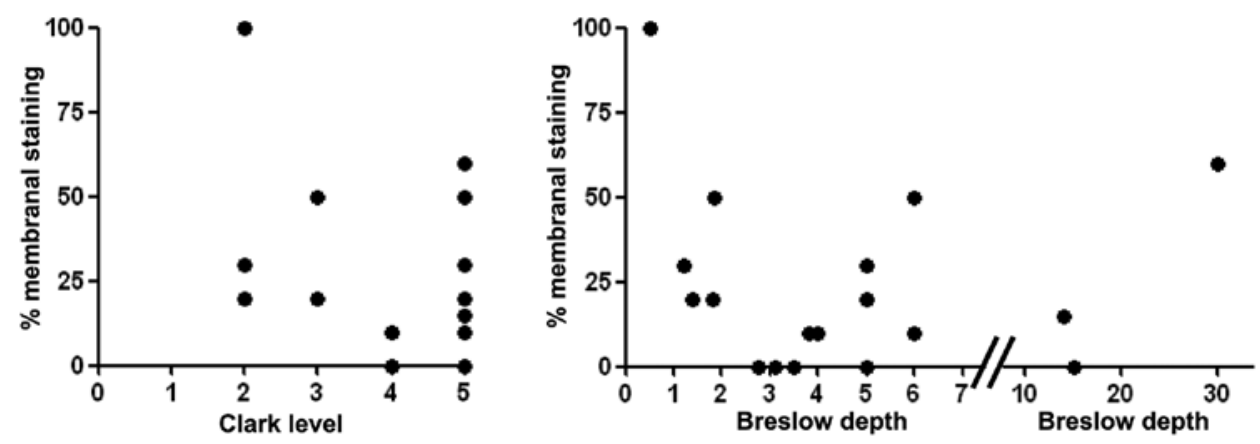

Figure 2. Membrane staining intensity vs. Clark and Breslow depth.
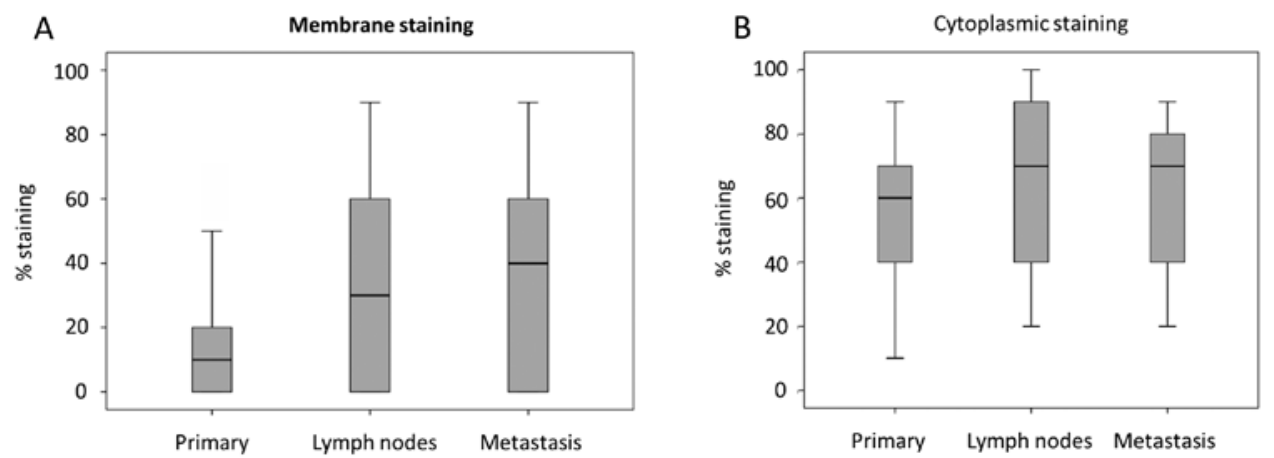

Figure 3. Membrane and cytoplasm staining with melanoma progression from primary to lymph node metastasis. Error bar, one standard deviation.

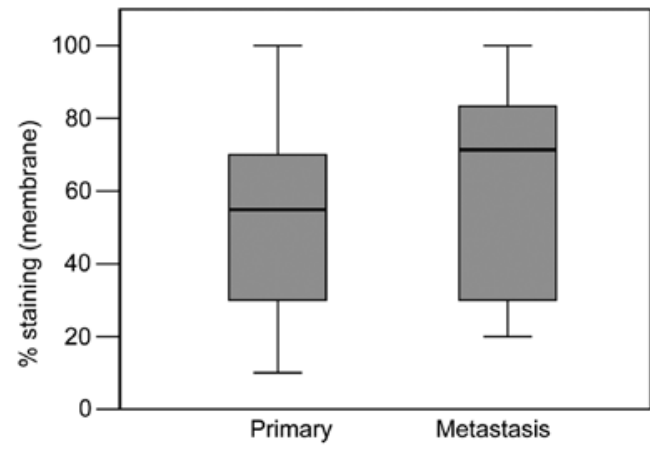

Figure 4. Membrane staining with melanoma progression from primary to metastasis. Error bar, one standard deviation.

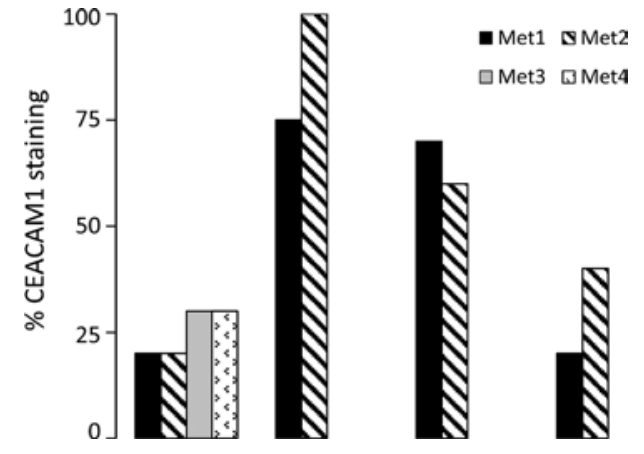

Figure 5. Membrane staining in patients with multiple metastasis.
For the cohort of 20 patients with primary and distant metastasis, a significant increase in membranal staining was noted, (Mann-Whitney test, $\mathrm{P}=0.026$ ) (Fig. 4) and again, no equivalent significant increase in cytoplasmic was observed. 

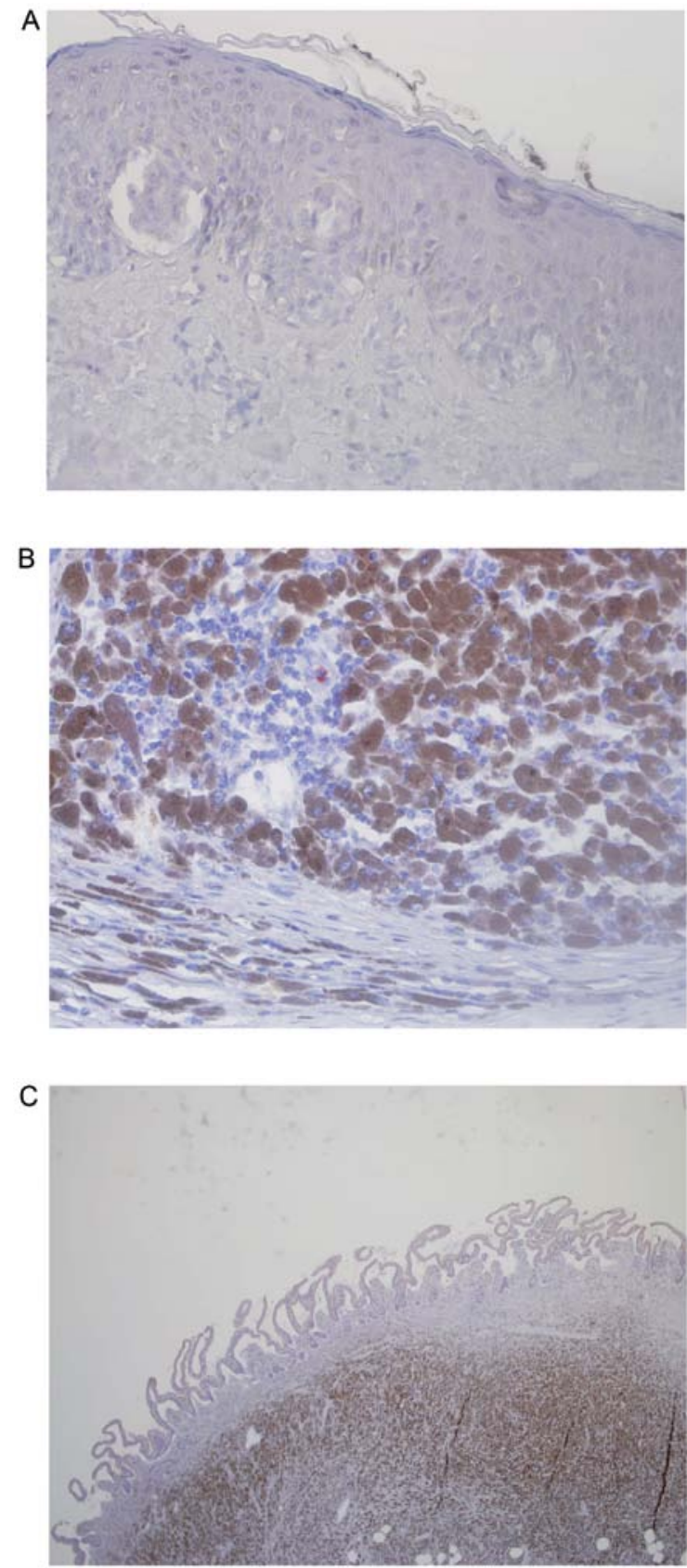

Figure 6. Micrographs of CEACAM1 slide preparations in an individual patient. (A) Primary cutaneous melanoma; very slight CEACAM1 staining. (B) Lymph node metastasis; moderate to high CEACAM1 staining. (C) Visceral (small bowel) metastasis; high CEACAM1 staining. CEACAM1, carcinoembryonic antigen-related cell adhesion molecule 1 .

In the 4 patients who had more than one metastatic lesion, no difference was found in the level of CEACAM1 membranal staining between the individual metastatic lesions (Fig. 5).

\section{Discussion}

Several studies have shown that normal melanocytes do not express CEACAM1 while most malignant melanoma lesions do express CEACAM1 $(10,20)$. We previously demonstrated that up to $89 \%$ of metastatic melanoma lesions are positive for CEACAM1 $(6,10)$ and that its expression increases concomitantly with tumor development and progression (10).
Furthermore, in an observational study by Thies et al that followed the outcome of 100 melanoma patients for 10 years, of the 40 patients with CEACAM1-positive primary melanoma, 28 patients developed metastatic disease as compared to only 6 of the 60 patients who were CEACAM1-negative (11). Taken together, this implies that CEACAM1 expression may be a crucial factor in the development of melanoma metastasis and poor prognosis. However, most of the currently available information on CEACAM1 expression in melanoma is derived from comparisons between patients and healthy individuals, or between isolated samples from various patients.

What makes the present study unique is that we were able to examine CEACAM1 expression in the same patient from primary tumor, to lymph node involvement, to distant metastasis, on a longitudinal axis. Importantly, as expected, CEACAM1 expression increased along the course of an individual's disease development and progression (Fig. 6A-C) There was a positive correlation between the percentage of cells stained for CEACAM1 and the intensity of staining. Notably, melanoma in situ specimens showed no CEACAM1 staining, implying that CEACAM1 expression is associated with the ability of the primary tumor to invade the basement membrane of the epidermis and subsequently metastasize. This conforms with Gambichler et al who found that CEACAM1 expression increases with progression from benign nevi to dyplastic nevi to invasive melanoma (21). This finding may enable a more accurate mapping of the timing of CEACAM1 expression during neoplastic progression. However, we did not find a correlation between CEACAM1 expression and the various prognostic indicators in melanoma such as primary lesion thickness, ulceration and lymphocytic infiltration.

We recently reported that CEACAM1 facilitates the proliferation of melanoma cells in a Sox-2-dependent manner (10). Additionally, melanoma cells utilize CEACAM1 as an immune evasion mechanism $(16,18,19)$. Moreover, we previously demonstrated that higher CEACAM1 expression levels improve the immune evasive phenotype (22). Therefore, it is conceivable that the rise in CEACAM1 expression along the disease progression of a patient contributes to its ability to proliferate and escape immune destruction to facilitate metastasis.

We previously reported on a monoclonal antibody blocking CEACAM1, which renders melanoma cells more vulnerable to natural killer cells and cytotoxic T cells (6). This strategy is analogous to the anti-PD-1 paradigm, and could be further developed as a novel drug. Multiple clinical trials have proven the efficacy of various immuno-oncology agents, such as antiCTLA-4 $(2,3)$ or anti-PD-1 (4), and initial positive data have even emerged in the adjuvant setting with anti-CTLA-4 (23). The present study may provide pertinent data for future development of CEACAM1-guided therapy. In addition, different metastatic lesions within the same patient exhibited similar CEACAM1 expression pattern (Fig. 5). This suggests that the different metastatic deposits benefit similarly by targeting CEACAM1. However, this issue should be further studied in a larger cohort.

There are many aspects of the function and expression of CEACAM1 which remain unknown. However, there may be valuable therapeutic consequences in understanding the mechanisms contributing to overexpression of CEACAM1. 
Malignant melanoma remains an extremely challenging problem for clinicians. Although at an early stage, melanoma is treated primarily with surgical extirpation, treatment of metastatic melanoma remains a profound clinical problem, as melanoma is resistant to most standard chemotherapy and radiation strategies. The use of immunotherapy in disseminated melanoma has increased dramatically with the addition of new immunotherapies to the physician's armamentarium, such as the anti-CTLA-4 agent ipilimumab, or adoptive cell transfer, in addition to the use of the cytokine interferon $\alpha$, all of which attempt to optimize the immunogenic response of the host to the disease. CEACAM1 represents a novel area of research which may have profound influence in future methods of harnessing cellular immunity to combat this disease.

There are limitations to the present study. The study cohort was small, and biased towards more aggressive lesions, and thus may not be representative of the entire spectrum of melanoma. It was undertaken to confirm our previous findings concerning the importance of CEACAM1 expression, within the same individual patient with disease progression. As CEACAM1 expression and potential blockade may offer a new horizon in immunotherapy, the results of the present study, combined with our previous studies, confirm that CEACAM1 is potentially an extremely useful target for arresting melanoma progression.

\section{References}

1. Sapoznik S, Hammer O, Ortenberg R, et al: Novel anti-melanoma immunotherapies: Disarming tumor escape mechanisms. Clin Dev Immunol 2012: 818214, 2012.

2. Hodi FS, O'Day SJ, McDermott DF, et al: Improved survival with ipilimumab in patients with metastatic melanoma. $\mathrm{N}$ Engl $\mathrm{J}$ Med 363: 711-723, 2010.

3. Robert C, Thomas L, Bondarenko I, et al: Ipilimumab plus dacarbazine for previously untreated metastatic melanoma. $\mathrm{N}$ Engl J Med 364: 2517-2526, 2011.

4. Hamid O, Robert C, Daud A, et al: Safety and tumor response with lambrolizumab (anti-PD-1) in melanoma. N Engl J Med 369: 134-144, 2013.

5. Brahmer JR, Tykodi SS, Chow LQ, et al: Safety and activity of anti-PD-L1 antibody in patients with advanced cancer. N Engl J Med 366: 2455-2465, 2012.

6. Ortenberg R, Sapir Y, Raz L et al: Novel immunotherapy for malignant melanoma with a monoclonal antibody that block CEACAM1 homophillic interactions. Mol Cancer Ther 6: 1300-1310, 2012.

7. Gray-Owen SD and Blumberg RS: CEACAM1: contact-dependent control of immunity. Nat Rev Immunol 6: 433-446, 2006.
8. Dango S, Sienel W, Schreiber M, et al: Elevated expression of carcinoembryonic antigen-related cell adhesion molecule 1 (CEACAM1) is associated with increased angiogenic potential in non small cell lung cancer. Lung Cancer 60: 426-433, 2008.

9. Zhou CJ, Liu B, Zhu KX, et al: The different expression of carcinoembryonic antigen-related cell adhesion molecule 1 (CEACAM1) and possible roles in gastric carcinoma. Pathol Res Pract 205: 483-489, 2009.

10. Ortenberg R, Galore-Haskel G, Greenberg I, et al: CEACAM1 promotes melanoma cell growth through Sox-2. Neoplasia 16: 451-460, 2014.

11. Thies A, Moll I, Berger J, et al: CEACAM1 expression in cutaneous malignant melanoma predicts the development of metastatic disease. J Clin Oncol 20: 2530-2536, 2002.

12. Laack E, Nikbakht H, Peters A, et al: Expression of CEACAM1 in adenocarcinoma of the lung: a factor of independent prognostic significance. J Clin Oncol 20: 4279-4284, 2002.

13. Sienel W, Dango S, Woelfle U, et al: Elevated expression of carcinoembryonic antigen-related cell adhesion molecule 1 promotes progression of non-small cell lung cancer. Clin Cancer Res 9: 2260-2266, 2003.

14. Muller MM, Klaile E, Vorontsova O, et al: Homophillic adhesion and CEACAM1-S regulate dimerization of CEACAM1-L and recruitment of SHP-2 and c-SRC. J Cell Biol 187: 569-581, 2009.

15. Chen Z, Chen L, Qiao SW, et al: Carcinoembryonic antigenrelated cell adhesion molecule 1 inhibits proximal TCR signaling by targeting ZAP-70. J Immunol 180: 6085-6093, 2008.

16. Markel G, Leiberman N, Katz G, et al: CD66a interactions between human melanoma and NK cells: a novel class I MHC-independent inhibitory mechanism of cytotoxicity. J Immunol 168: 2803-2810, 2002.

17. Markel G, Wolf D, Hanna J, Gazit R, et al: Pivotal role of CEACAM1 protein in the inhibition of activated decidual lymphocyte functions. J Clin Invest 110: 943-953, 2002.

18. Markel G, Seidman R, Stern N, et al: Inhibition of human tumorinfiltrating lymphocyte effector functions by the homophillic carcinoembryonic cell adhesion molecules 1 interactions. J Immunol 177: 6062-6071, 2006.

19. Markel G, Ortenberg R, Seidman R, et al: Systemic dysregulation of CEACAM1 in melanoma patients. Cancer Immunol Immmunother 59: 215-230, 2010.

20. Ebrahimnejad A, Streichert T, Nollau P, et al: CEACAM1 enhances invasion and migration of melanocytic and melanoma cells. Am J Pathol 165: 1781-1787, 2004.

21. Gamblicher T, Grothe S, Rotterdam S, et al: Protein expression of carcinoembryonic antigen cell adhesion molecules in benign and malignant melanocytic skin lesions. Am J Clin Pathol 131: 782-787, 2009.

22. Markel G, Seidman R, Cohen Y, et al: Dynamic expression of protective CEACAM1 on melanoma cells during specific immune attack. Immunology 126: 186-200, 2009.

23. Eggermont AM, Chiarion-Seleni V, Grob JJ, et al: Ipilimumab versus placebo after complete resection of stage III melanoma: initial efficacy and safety results from the EORTC 18071 phase III trial. Presented at the 2014 Annual Meeting of the American Society of Clinical Oncology; May 30-June 2, 2014; Chicago, IL. J Clin Oncol 32 (15 Suppl): LBA9008, 2014. 